\title{
Shotgun Sequencing
}

National Cancer Institute

\section{Source}

National Cancer Institute. Shotgun Sequencing. NCI Thesaurus. Code C19646.

Unordered sequencing. Unmapped clones spanning a region of interest are sequenced and then assembled into a continuous sequence. 